\title{
EDITORIAL
}

\section{Cancer: Challenges and Remedies}

\author{
*ME Hoque \\ ${ }^{1}$ Prof. Md. Ehteshamul Haque, Professor(CC), Department of Radiation Oncology \\ Anwer Khan Modern Medical College \& Hospital (AKMMC\&H), Dhaka, Bangladesh \\ *Corresponding Author
}

\begin{abstract}
'Cancer' now-a-days become a common problem worldwide. World wide mortality in cancer stands as second, cardiovascular disease being the number one $^{1}$.Cancer can be defined as a cellular disorder characterized by progressive accumulation of a mass of cells, as a result of excessive production of cells, not compensated by appropriate cell loss; these cells progressively invade and damage the tissue and organ of the host ${ }^{2}$. Lack of awareness and negligence may increase the burden to the family and society at large. It is a disease for a person but a problem for the family, society \& nation as well as for the globe. This diseases burdens a symbol of social disaster for the family both for health \& economic point of view. A person and a family should be aware of such an unpredictable non-communicable disease problem due to its high treatment cost \& long term sufferings. It can be prevented, detected \& treated adequately if individuals are well aware of the risk factors, food habits, and life styles. The paper highlights global and local burden, challenges and remedial measures available in Bangladesh at this moment.
\end{abstract}

Cancer is a leading disease burden worldwide. An estimated 12.7 million new cancer cases occurred in 2008 of which 5.6 million occurred in economically developed countries and 7.1 million in economically developing countries. The corresponding estimates for total cancer deaths in 2008 were 7.6 million (about 21, 000 cancer deaths a day), 2.8 million in economically developed countries and 4.8 million in economically developing countries ${ }^{3}$. The common site of cancer is in lung, female breast, colorectal and stomach that accounted for $40 \%$ of all cases diagnosed worldwide. In men, lung cancer was the most common cancer (16.5\% of all new cases in men). Breast cancer was by far the most common cancer diagnosed in women $(23 \%$ of all new cases in women $)^{4}$. It is a leading cause of
7.6 million deaths (around 13\% of all deaths) in 2008. Half of all cancer deaths each year are due to lung, stomach, liver, colorectal and female breast cancers. In Bangladesh about 2 lakh people suffer from cancer every year of which 1.5 lakh dies. Out of that about $57 \%$ are male \& $43 \%$ are female. The most common cancer found in male is lung cancer $(25.5 \%) \&$ in female beast cancer $(25.6 \%)$. In addition, cancer in colon, head \& neck, esophagus, liver, cervix, prostate, pancreas, various gland tumors are also common. The incidence of cancer cervix is also alarming for the females. If the recent trend in major cancers remains some globally in the future, the burden of cancer will increase to 22 million new cases each year by 2030 . This represents an increase of $75 \%$ compared to that with 2008 .

Certain challenge like; smoking habit, use of tobacco, food habit specially the fast food, life style, infections, and environmental pollution needs careful attention to minimize the effect of those probable factors. In a study on risk factors and common cancers in Bangladesh found important risk factors in men are smoking $(68.14 \%)$, Betal leaf $(67.55 \%)$ while in women recurrent bacterial virae diseases $(39.10 \%)$ and obesity (37\%) chronic disease $(37.03 \%)^{5}$. In many cases cause of cancer is unknown therefore; avoidance of risk factors, food habits \& life style modification including food safety issues needs careful consideration both at individual, social, and political level. There is a great concern about environmental pollution \& legislation measures, improvement of those can help minimizing this burden too. There is an urgent need for resource mobilization for early detection of cancer by surveillance \& screening among the population at risk using primary health care network existing in this country.

Personal plan for regular health check-up for early detection \& treatment by the specialist can help minimizing its burden in addition to minimize 
treatment cost too. 'Health Insurance' can also be considered like other countries to avoid economic burden for the family. In addition, social awareness may help a lot involving social workers, policy makers, and media (both print $\&$ electronic) to work on it together. Now there are enough answers for the solution for cancer.

Now a day's cancer can be detected earlier and can be cured by radiotherapy, chemotherapy, hormonal therapy, targeted therapy, bone marrow transplantation and facilities are well established in our country6.7. There are palliative care departments in various canters for cancer patients that have started working combined with multidisciplinary experts. PET CT \& latest true beam machine, anti-cancer drugs are also available for cancer treatment. Technological innovations in this field added up opportunities in the prevention, detection, and treatment towards minimizing cure rate and mortality rate.

However the most important aspect is community approach preventive measures like adequate physical work, avoid chunk and fast food, soft drink, tobacco smoking, betel nut \& leaf avoidance, alcohol abstinence, timely taking vaccination, maintain proper body weight according to BMI, genetic and familial screening are essential to combed cancer.

The technological innovation in the treatment of cancer patient has opened much opportunity to deal it efficient \& effectively if detected early. Professionals in the discipline particularly oncologists are trying hard to overcome the obstacles related to this service. Treatment modalities \& facilities, diagnostic procedures, automated machines, latest research drugs and advance radiotherapy machines all these are making the cancer treatment more easy and realistic. Anwer Khan Modern Medical College \& Hospital (AKIMMC\&H) has undertaken the steps to address those challenges of minimizing the burden of cancer cases as a part of social accountability \& responsibility by creating opportunities at an affordable cost. The institution is planning to establish a modern 'Cancer Care Center' with all the lastest facilities including latest technologies, chemotherapy, radiotherapy, targeted therapy, surgery, and palliative care, Hopefully establishment of a modern 'Cancer Care Center' with all the latest facilities mentioned will lead to define better strategies \& management of cancer in Bangladesh and will offer a bright future towards prolonging life for the cancer patients in this country.

\section{References}

1. Pathologic Basis of Diseases. Editor Kumar V, Abbas AK, Fausto N, Aster JC, 8th Edition 2010, Publisher Saunders Elsevier P 260, 272

2. Manual of clinical oncology: 7th Edition; Chapter1: Principles of Definition.

3. GLOBOCAN 2008: Global Cancer facts \& figure, 2nd Edition.

4. Cancer Registry Report: National Institute of Cancer Research and Hospital, 2005-2007.

5. Cancer Research UK: World cancer fact sheet, August 2012.

6. Shahriar M, Islam RB, Mahmood As Mamun MSA, Nahar SS, Sadiana T, Shahid S. 2011 S.J. Pharm, Sci; 4 (2) $35-41$.

7. Websites: www.cancerresearchuk.org, www.uicc.org, www. nicrhbd.org. 